\title{
ON THE DEGREE OF REPEATED RADICAL EXTENSIONS
}

\author{
FERNANDO SZECHTMAN
}

This paper is dedicated to Natalio H. Guersenzvaig.

\begin{abstract}
We answer a question posed by Mordell in 1953, in the case repeated radical extensions, and find necessary and sufficient conditions for $\left[F\left[\sqrt[m_{1}]{N_{1}}, \ldots, \sqrt[m_{\ell}]{N_{\ell}}\right]: F\right]=m_{1} \cdots m_{\ell}$, where $F$ is an arbitrary field of characteristic not dividing any $m_{i}$.
\end{abstract}

\section{INTRODUCTION}

We fix throughout a unique factorization domain $D$ with field of fractions $F$, allowing for the possibility that $D=F$, and write $c(F)$ for the characteristic of $F$. We also fix $\ell \in \mathbb{N}$, $m_{1}, \ldots, m_{\ell} \in \mathbb{N}, m=\operatorname{lcm}\left\{m_{1}, \ldots, m_{\ell}\right\}$, and $N_{1}, \ldots, N_{\ell} \in D$. A prime means a prime positive integer.

In this paper, we give a necessary and sufficient condition for

$$
\left[F\left[\sqrt[m_{1}]{N_{1}}, \ldots, \sqrt[m_{\ell}]{N_{\ell}}\right]: F\right]=m_{1} \cdots m_{\ell}
$$

assuming only $c(F) \nmid m$. This settles a problem posed by Mordell $[\mathrm{M}]$ in 1953, in the case of repeated radical extensions.

The degrees of repeated radical extensions have been studied by several authors, including

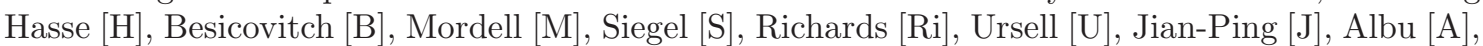
and Carr and O'Sullivan CS.

The question of when $[F[\sqrt[n]{a}]: F]=n$ was solved by Vahlen $[\mathrm{V}]$ in 1895 if $F=\mathbb{Q}$, Capelli $[\mathrm{C}]$ in 1897 if $F$ has characteristic 0, and Rédei [R, Theorem 428] in 1959 in general.

Irreducibility Criterion (C). The polynomial $X^{n}-a \in F[X]$ is irreducible if and only if $a \notin F^{p}$ for every prime factor $p$ of $n$, and if $4 \mid n$ then $a \notin-4 F^{4}$.

In particular, if $-a \notin F^{2}$ and $a \notin F^{p}$ for every prime factor $p$ of $n$, then $X^{n}-a$ is irreducible. The special case of $(\mathrm{C})$ when $n$ is prime is due to Abel; a very simple proof of this case can be found in [R, Theorem 427].

Provided $F$ contains a primitive $m$ th root of unity, Hasse [H] showed that (1D) holds if and only if

$$
\left(\sqrt[m_{1}]{N_{1}}\right)^{a_{1}} \times \cdots \times\left(\sqrt[m_{\ell}]{N_{\ell}}\right)^{a_{\ell}} \in F, a_{i} \geq 0 \text {, only when } m_{1}\left|a_{1}, \ldots, m_{\ell}\right| a_{\ell} .
$$

Later, Besicovitch [B] proved (11) assuming: $D=\mathbb{Z}$; each $N_{i}$ is positive and has a prime factor that divides it only once and does not divide any other $N_{j}$; each $\sqrt[m_{i}]{N_{i}}$ is positive and real (the $m_{1} \cdots m_{\ell}$ embeddings of $\mathbb{Q}\left[\sqrt[m_{1}]{N_{1}}, \ldots, \sqrt[m_{\ell}]{N_{\ell}}\right]$ into $\mathbb{C}$ then yield (10) for all other $m_{i}$ th roots of the $N_{i}$ ). The special case of Besicovitch's result when $m_{1}=\cdots=m_{\ell}$ and every $N_{i}$ is prime appears in Richards [Ri] (for the more elementary case $m_{1}=\cdots=m_{\ell}=2$, see [F, Ro ). Assuming that $N_{1}, \ldots, N_{\ell}$ are pairwise relatively prime, Ursell [U] obtained a variation of Besicovitch's theorem.

2010 Mathematics Subject Classification. 12F05, 12F10, $12 \mathrm{E} 05$.

Key words and phrases. Radical extension, Galois extension, Vahlen-Capelli irreducibility criterion.

This research was partially supported by an NSERC grant. 
Mordell M] combined and extended the results of Hasse and Besicovitch, and proved (1) assuming (2), and that $F$ contains a primitive $m$ th root of unity or that $F$ is a subfield of $\mathbb{R}$ with all $\sqrt[m_{1}]{N_{1}}, \ldots, \sqrt[m_{\ell}]{N_{\ell}}$ real. In the latter case, all $N_{i}$ such that $m_{i}$ is even must be positive. Siegel [S] gave a theoretical description of the value of $\left[F\left[\sqrt[m_{1}]{N_{1}}, \ldots, \sqrt[m_{\ell}]{N_{\ell}}\right]: F\right]$ under Mordell's condition that $F$ be a subfield of $\mathbb{R}$ with all $\sqrt[m_{i}]{N_{i}}$ real. Albu $\mathrm{A}$ extended the work of Mordell and Siegel to the case when $F$ contains a primitive $m$ th root of unity or all $m$ th roots of unity in $F\left[\sqrt[m_{1}]{N_{1}}, \ldots, \sqrt[m_{\ell}]{N_{\ell}}\right]$ belong to $\{1,-1\}$. Under these weaker assumptions, (11) is still shown in A to be a consequence of (2). It is worth noting that, except for (2), none of the aforementioned conditions are necessary for (11) to hold. A different approach was taken by Jian-Ping [J], using valuation theory, when $F$ is an algebraic number field; he succeeded in avoiding any assumptions on roots of unity and proved a more general version of (1), applicable to repeated extensions via Eisenstein polynomials, not just binomials. Nevertheless, Jian-Ping's hypotheses are also unnecessary for (1) to hold. Indeed, when the ring of integers of $F$ is a UFD, each $N_{i}$ is forced to have an irreducible factor that divides it only once and does not divide any other $N_{j}$. More recently, Carr and O'Sullivan [CO proved a fairly general result on the linear independence of roots and reproved Mordell's theorem as an application.

Set $J=\{1, \ldots, \ell\}, \mathcal{P}=\{p \mid p$ is a prime factor of $m\}$, and for each $i \in J$ and $p \in \mathcal{P}$ let $m_{i}(p)$ be the $p$-part of $m_{i}$, so that $m_{i}(p)=p^{n_{i}}$, where $n_{i} \geq 0, p^{n_{i}} \mid m_{i}$ and $p^{n_{i}+1} \nmid m_{i}$. It is clear that

$$
\left[F\left[\sqrt[m_{1}]{N_{1}}, \ldots, \sqrt[m_{\ell}]{N_{\ell}}\right]: F\right]=m_{1} \cdots m_{\ell} \Leftrightarrow\left[F\left[\sqrt[m_{1}(p)]{N_{1}}, \ldots, \sqrt[m_{\ell}(p)]{N_{\ell}}\right]: F\right]=m_{1}(p) \cdots m_{\ell}(p)
$$

for all $p \in \mathcal{P}$. We are thus reduced to study the case when each $m_{i}=m_{i}(p)$ for a fixed prime $p$. We split this case in two subcases depending on the parity of $p$. For each prime $p$, we set

$$
\mathcal{S}_{p}=\left\{N_{1}, N_{1}^{e_{1}} N_{2}, N_{1}^{e_{1}} N_{2}^{e_{2}} N_{3}, \ldots, N_{1}^{e_{1}} \cdots N_{\ell-1}^{e_{\ell-1}} N_{\ell}, 0 \leq e_{i}<p\right\} .
$$

In particular, $\mathcal{S}_{2}$ consists of all $N_{1}^{e_{1}} \cdots N_{\ell}^{e_{\ell}}$ such that $e_{i} \in\{0,1\}$ and $\left(e_{1}, \ldots, e_{\ell}\right) \neq(0, \ldots, 0)$.

Theorem A. Let $n_{1}, \ldots, n_{\ell} \in \mathbb{N}, p$ an odd prime such that $c(F) \neq p$, and suppose $m_{i}=p^{n_{i}}$ for all $i \in J$. Then (1) holds if and only if $\mathcal{S}_{p} \cap D^{p}=\emptyset$.

The well-known example $[\mathbb{Q}[\sqrt[4]{-1}, \sqrt[4]{2}]: \mathbb{Q}]=8$ shows that Theorem A fails if $p=2$. The above criteria impose general conditions that disallow this example. Close examination of numerous pathological cases led us to the exact conditions required when $p=2$. We say that $\left(N_{1}, \ldots, N_{\ell}\right)$ is 2-defective if the following two conditions hold: $\mathcal{S}_{2} \cap D^{2}=\emptyset$ but $\mathcal{S}_{2} \cap\left(-D^{2}\right) \neq \emptyset$ (this readily implies that $\left|\mathcal{S}_{2} \cap\left(-D^{2}\right)\right|=1$, as shown in Lemma 5); if $-d^{2}=M=N_{1}^{f_{1}} \cdots N_{\ell}^{f_{\ell}}$ is the only element of $\mathcal{S}_{2} \cap\left(-D^{2}\right)$, where $d \in D, 0 \leq f_{i}<2$, and $M^{\sharp}=\left\{i \in J \mid f_{i}=1\right\}$ is nonempty (since $\mathcal{S}_{2} \cap D^{2}=\emptyset$, the exponents $f_{i}$ are uniquely determined by $M$, whence $M^{\sharp}$ is well-defined), then $4 \mid m_{i}$ for all $i \in M^{\sharp}$, and if $i \in M^{\sharp}$, then

$$
\pm 2 d \prod_{j \neq i} N_{j}^{e_{j}} \in D^{2} \text { for some choice of } 0 \leq e_{j}<1
$$

Since $-M=N_{1}^{f_{1}} \cdots N_{\ell}^{f_{\ell}} \in D^{2}$, the outcome of (4) is independent of the actual choice of $i \in M^{\sharp}$.

Theorem B. Let $n_{1}, \ldots, n_{\ell} \in \mathbb{N}$ and suppose that $c(F) \neq 2$ and $m_{i}=2^{n_{i}}$ for all $i \in J$. Then (1) holds if and only if $\mathcal{S}_{2} \cap D^{2}=\emptyset$ and $\left(N_{1}, \ldots, N_{\ell}\right)$ is not 2-defective.

Combining (3) with Theorems A and B we immediately obtain a general criterion for (1). This requires additional notation. For each $p \in \mathcal{P}$, we set $J(p)=\left\{i \mid i \in J\right.$ and $\left.p \mid m_{i}\right\}$, and write

$$
\begin{gathered}
J(p)=\{i(p, 1), \ldots, i(p, \ell(p))\}, \quad i(p, 1)<\cdots<i(p, \ell(p)) \\
\mathcal{S}(p)=\left\{N_{i(p, 1)}, N_{i(p, 1)}^{e_{1}} N_{i(p, 2)}, N_{i(p, 1)}^{e_{1}} N_{i(p, 2)}^{e_{2}} N_{i(p, 3)}, \ldots, N_{i(p, 1)}^{e_{1}} \cdots N_{i(p, \ell(p)-1)}^{e_{\ell(p)-1}} N_{i(p, \ell(p))}, 0 \leq e_{j}<p\right\} .
\end{gathered}
$$


Theorem C. Suppose that $c(F) \neq m$. Then (1) holds if and only if $\mathcal{S}(p) \cap D^{p}=\emptyset$ for every $p \in \mathcal{P}$ and, if $2 \in \mathcal{P}$, then $\left(N_{i(2,1)}, \ldots, N_{i(2, \ell(2))}\right)$ is not 2-defective.

The next example illustrates the use of Theorems B and C, and lies outside of the scope of the aforementioned criteria.

Example 1. Suppose that $-1 \notin F^{2}$ and each of $A, B, C \in D$ has an irreducible factor that divides it only once and does not divide any of the two other elements. Then

$$
\left[F\left[\sqrt[m_{1}]{A B}, \sqrt[m_{2}]{B C}, \sqrt[m_{3}]{-C A}\right]: F\right]=m_{1} m_{2} m_{3}
$$

if and only if at least one of $m_{1}, m_{2}, m_{3}$ is not divisible by 4 or none of $\pm 2 A, \pm 2 B, \pm 2 C \in D^{2}$.

As we are dealing with a classical and basic problem, we purposely resort to elementary and complete arguments in order maximize the potential readership of our solution.

\section{LEMMATA}

Given a nonzero $a \in F$ we write $\langle a\rangle$ for the subgroup of $F^{\times}$generated by $a$.

Lemma 1. Let $p$ be a prime such that $c(F) \neq p$ and suppose $b_{1}, \ldots, b_{n} \in F$ are nonzero. Then

$$
F\left[\sqrt[p]{b_{1}}, \ldots, \sqrt[p]{b_{n}}\right]^{p} \cap F=F^{p}\left\langle b_{1}, \ldots, b_{n}\right\rangle .
$$

Proof. Let $M, N \in F$ be nonzero. We claim that if $\sqrt[p]{M} \in F[\sqrt[p]{N}]$ then $M \in F^{p}\langle N\rangle$. This is clear if $N \in F^{p}$ so we assume $N \notin F^{p}$.

Set $K=F[\zeta]$, where $\zeta$ is a primitive $p$ th root of unity. Then $K / F$ is a Galois extension with Galois group isomorphic to a subgroup of $(\mathbb{Z} / p \mathbb{Z})^{\times}$. In particular, $[K: F]$ divides $(p-1)$. It follows that $K^{p} \cap F=F^{p}$. Indeed, suppose $a \in F$ and $\alpha \in K$ satisfies $\alpha^{p}=a$. Since $[F[\alpha]: F]$ divides $[K: F]$, it also divides $p-1$. As $p \nmid(p-1), X^{p}-a \in F[X]$ is reducible, whence $a \in F^{p}$ by $(\mathrm{C})$.

By assumption, $N \notin F^{p}$. Thus $N \notin K^{p}$ as indicated above, so $X^{p}-N \in K[X]$ is irreducible by (C). Thus $\left\{1, \sqrt[p]{N}, \ldots, \sqrt[p]{N^{p-1}}\right\}$ is a $K$-basis of $K[\sqrt[p]{N}]$. By assumption, $\sqrt[p]{M} \in K[\sqrt[p]{N}]$, so

$$
\sqrt[p]{M}=a_{0}+a_{1} \sqrt[p]{N}+\cdots+a_{p-1} \sqrt[p]{N^{p-1}}, \quad a_{i} \in K
$$

Note that $K[\sqrt[p]{N}] / K$ is a Galois extension with cyclic Galois group $\langle\sigma\rangle$, where $\sigma(\sqrt[p]{N})=\zeta \sqrt[p]{N}$. Since $\sqrt[p]{M}$ is a root of $X^{p}-M$, we must have $\sigma(\sqrt[p]{M})=\zeta \sqrt[p]{M}$ for some $0 \leq i<p$. Applying $\sigma$ to (6), we obtain

$$
\zeta^{i} \sqrt[p]{M}=a_{0}+a_{1} \zeta \sqrt[p]{N}+\cdots+a_{p-1} \zeta^{p-1} \sqrt[p]{N^{p-1}}
$$

On the other hand, multiplying (6) by $\zeta^{i}$ yields

$$
\zeta^{i} \sqrt[p]{M}=a_{0} \zeta^{i}+a_{1} \zeta^{i p p} \sqrt{N}+\cdots+a_{p-1} \zeta^{i p} \sqrt{N^{p-1}}
$$

From the $K$-linear independence of $1, \sqrt[p]{N}, \ldots, \sqrt[p]{N^{p-1}}$ we infer that $a_{j}=0$ for all $j \neq i$. Thus

$$
\sqrt[p]{M}=a \sqrt[p]{N^{i}}, \quad a \in K
$$

whence $M=a^{p} N^{i}$. Thus $M / N^{-i} \in K^{p} \cap F=F^{p}$, so $M \in F^{p}\langle N\rangle$.

By above, $F\left[\sqrt[p]{b_{1}}\right]^{p} \cap F=F^{p}\left\langle b_{1}\right\rangle$. Suppose $n>1$ and $F\left[\sqrt[p]{b_{1}}, \ldots, \sqrt[p]{b_{n-1}}\right]^{p} \cap F=F^{p}\left\langle b_{1}, \ldots, b_{n-1}\right\rangle$. Then

$$
\begin{aligned}
F\left[\sqrt[p]{b_{1}}, \ldots, \sqrt[p]{b_{n-1}}, \sqrt[p]{b_{n}}\right]^{p} \cap F & =\left(F\left[\sqrt[p]{b_{1}}, \ldots, \sqrt[p]{b_{n-1}}\right]\left[\sqrt[p]{b_{n}}\right]\right)^{p} \cap F\left[\sqrt[p]{b_{1}}, \ldots, \sqrt[p]{b_{n-1}}\right] \cap F \\
& =F\left[\sqrt[p]{b_{1}}, \ldots, \sqrt[p]{b_{n-1}}\right]^{p}\left\langle b_{n}\right\rangle \cap F
\end{aligned}
$$

Let $\alpha \in F\left[\sqrt[p]{b_{1}}, \ldots, \sqrt[p]{b_{n-1}}\right]^{p}\left\langle b_{n}\right\rangle \cap F$. Then $\alpha \in F$ and $\alpha b_{n}^{i} \in F\left[\sqrt[p]{b_{1}}, \ldots, \sqrt[p]{b_{n-1}}\right]^{p} \cap F$ for some $i \in \mathbb{Z}$. Thus $\alpha b_{n}^{i} \in F^{p}\left\langle b_{1}, \ldots, b_{n-1}\right\rangle$ and therefore $\alpha \in F^{p}\left\langle b_{1}, \ldots, b_{n-1}, b_{n}\right\rangle$. 
Lemma 2. Suppose $-1 \notin F^{2}$ and $\pm a \notin F^{2}$. Then for any $n \in \mathbb{N}$, we have $\sqrt{-1} \notin F\left[\sqrt[2^{n}]{a}\right]$.

Proof. We show by induction that $\sqrt{-1} \notin F[\sqrt[2 n]{a}]$ and $\pm \sqrt[2^{n}]{a} \notin F[\sqrt[2 n]{a}]^{2}$. The fact that $\sqrt{-1} \notin F[\sqrt{a}]$ follows from Lemma 1. Suppose, if possible, that $\pm \sqrt{a}=z^{2}$, where $z \in F[\sqrt{a}]$. Then $z=x+y \sqrt{a}$, where $x, y \in F$, so that $\pm \sqrt{a}=x^{2}+a y^{2}+2 x y \sqrt{a}$. It follows that $x^{2}+a y^{2}=0$. As $-a \notin F^{2}$, we infer $x=y=0$, a contradiction.

Assume we have shown that $\sqrt{-1} \notin F[\sqrt[2 n]{a}]$ and $\pm \sqrt[2^{n}]{a} \notin F\left[\sqrt[2^{n}]{a}\right]^{2}$ for some $n \in \mathbb{N}$. Since $\pm a \notin F^{2}$, (C) implies $\left[F\left[\sqrt[2^{n}]{a}\right]: F\right]=2^{n}$ and $\left[F\left[\sqrt[2^{n+1}]{a}\right]: F\right]=2^{n+1}$. But $\sqrt{-1} \notin F\left[\sqrt[2^{n}]{a}\right]$, so $F\left[\sqrt[2^{n+1}]{a}\right]=F\left[\sqrt[2^{n}]{a}, \sqrt{-1}\right]$. Thus $\sqrt[2^{n+1}]{a}=\alpha+\beta \sqrt{-1}$ for unique $\alpha, \beta \in F\left[\sqrt[2^{n}]{a}\right]$. Squaring, we get $\sqrt[2^{n}]{a}=\alpha^{2}-\beta^{2}+2 \alpha \beta \sqrt{-1}$, which implies $\alpha \beta=0$ and $\alpha^{2}-\beta^{2}=\sqrt[2^{n}]{a}$, a contradiction.

Lemma 3. Suppose $c(F) \neq 2$, let $n \in \mathbb{N}$, and set $K=F[\zeta]$, where $\zeta$ is a primitive $2^{n}$ th root of unity. If $n \leq 2$ or $-1 \in F^{2}$, then $G=\operatorname{Gal}(K / F)$ is cyclic.

Proof. We have an embedding $\Psi: G \rightarrow\left(\mathbb{Z} / 2^{n} \mathbb{Z}\right)^{\times}, \sigma \rightarrow[s]$, where $\sigma(\zeta)=\zeta^{s}$. This settles the case $n \leq 2$. Assume henceforth that $n \geq 3$. We have $\left(\mathbb{Z} / 2^{n} \mathbb{Z}\right)^{\times}=\langle a, b\rangle$, where $a=[5], b=[-1]$ and $\langle a\rangle \cap\langle b\rangle$ is trivial [Vi, Chapter VI]. By hypothesis, $-1=\alpha^{2}$, where $\alpha \in F \cap\langle\zeta\rangle$. Suppose, if possible, that $b \in \Psi(G)$, say $b=\Psi(\sigma)$. Then $\sigma(\alpha)=\alpha^{-1}=-\alpha$, since $\alpha$ is a power of $\zeta$, and $\sigma(\alpha)=\alpha$, since $\alpha \in F$. This contradiction shows that $b \notin \Psi(G)$. Now any subgroup $S$ of $\langle a, b\rangle$ that does not contain $b$ must be cyclic (if $S$ is not trivial, it is generated by $a^{i}$ or $a^{i} b$, where $i$ is the smallest positive integer such that an element of this type is in $S$ ). Thus $G$ is cyclic.

Lemma 4. Let $n \in \mathbb{N}, p$ an odd prime such that $c(F) \neq p$, and set $K=F[\zeta]$, where $\zeta$ is a primitive $p^{n}$ th root of unity. Then $\operatorname{Gal}(K / F)$ is cyclic.

Proof. $\operatorname{Gal}(K / F)$ is isomorphic to a subgroup of $\left(\mathbb{Z} / p^{n} \mathbb{Z}\right)^{\times}$, which is a cyclic group.

Lemma 5. Suppose $\mathcal{S}_{2} \cap D^{2}=\emptyset$. Then $\left|S_{2} \cap\left(-D^{2}\right)\right| \leq 1$, with $\left|S_{2} \cap\left(-D^{2}\right)\right|=0$ if $-1 \in F^{2}$.

Proof. Suppose $M \neq N$ are in $\mathcal{S}_{2} \cap\left(-D^{2}\right)$. Then $M N \in D^{2}$ and $M N=e^{2} P$, where $P \in \mathcal{S}_{2}$ and $e \in D$. Thus $P \in D^{2}$, against $\mathcal{S}_{2} \cap D^{2}=\emptyset$. If $-1 \in F^{2}$ then $-D^{2}=D^{2}$, so $\mathcal{S}_{2} \cap\left(-D^{2}\right)=\emptyset$.

Lemma 6. Suppose $c(F) \neq 2$, let $n \in \mathbb{N}$ and set $K=F[\zeta]$, where $\zeta$ is a primitive $2^{n}$ th root of unity. Assume $\mathcal{S}_{2} \cap D^{2}=\emptyset$. Then $\left|\mathcal{S}_{2} \cap K^{2}\right| \in\{0,1,3\}$. Moreover, if $\left|\mathcal{S}_{2} \cap K^{2}\right|=3$ then one of the elements of $\mathcal{S}_{2} \cap K^{2}$ is in $\mathcal{S}_{2} \cap\left(-D^{2}\right)$, and we have $-1 \notin F^{2}, n \geq 3$.

Proof. Suppose $M \neq N$ are in $\mathcal{S}_{2} \cap K^{2}$. Then $M N \in K^{2}$ and $M N=e^{2} P$, where $P \in \mathcal{S}_{2}$ and $e \in D$, so $P \in D^{2}$. Lemma 1 implies that $F[\sqrt{M}], F[\sqrt{N}], F[\sqrt{P}]$ are distinct intermediate subfields of $K / F$ of degree 2. In particular, $\operatorname{Gal}(K / F)$ is not cyclic. Now $\operatorname{Gal}(K / F)$ is isomorphic to a subgroup of $\left(\mathbb{Z} / 2^{n} \mathbb{Z}\right)^{\times}$, so $n \geq 3$ and $\left(\mathbb{Z} / 2^{n} \mathbb{Z}\right)^{\times} \cong\left(\mathbb{Z} / 2^{n-2} \mathbb{Z}\right) \times(\mathbb{Z} / 2 \mathbb{Z})$. Any subgroup of $\left(\mathbb{Z} / 2^{n-2} \mathbb{Z}\right) \times(\mathbb{Z} / 2 \mathbb{Z})$ has at most 3 subgroups of index 2 , so the Galois correspondence implies that any intermediate subfield of $K / F$ of degree 2 must be equal to one of $F[\sqrt{M}], F[\sqrt{N}], F[\sqrt{P}]$. Lemma 1 readily implies that no element from $\mathcal{S}_{2}$ different from $M, N, P$ is in $K^{2}$. By Lemma 3 , $-1 \notin F^{2}$, so $F[\sqrt{-1}]$ must be equal to one of $F[\sqrt{M}], F[\sqrt{N}], F[\sqrt{P}]$, and Lemma 1 implies that one of $M, N, P$ is in $-D^{2}$.

Lemma 7. Let $n_{1}, \ldots, n_{\ell} \in \mathbb{N}$ and $p$ a prime such that $c(F) \neq p$ and $m_{i}=p^{n_{i}}$ for all $i \in J$. Let $K=F[\zeta]$, where $\zeta$ is a primitive $m$ th root of unity, $m=\operatorname{lcm}\left\{m_{1}, \ldots, m_{\ell}\right\}$. Suppose that $\mathcal{S}_{p} \cap K^{p}=\emptyset$. Then $\left[K\left[\sqrt[m_{1}]{N_{1}}, \ldots, \sqrt[m_{\ell}]{N_{\ell}}\right]: K\right]=m_{1} \cdots m_{\ell}$. 
Proof. By assumption $N_{1} \notin K^{p}$. Moreover, if $4 \mid m_{1}$ then $-1 \in K^{2}$ and therefore $-N_{1} \notin K^{2}$. It follows from $(\mathrm{C})$ that $\left[K\left[\sqrt[m_{1}]{N_{1}}\right]: K\right]=m_{1}$. Suppose $\left[K\left[\sqrt[m_{1}]{N_{1}}, \ldots, \sqrt[m_{i}]{N_{i}}\right]: K\right]=m_{1} \cdots m_{i}$ for some $1 \leq i<\ell$.

Assume, if possible, that $\sqrt[p]{N_{i+1}} \in K\left[\sqrt[m_{1}]{N_{1}}, \ldots, \sqrt[m_{i}]{N_{i}}\right]$. Then $K\left[\sqrt[p]{N_{i+1}}\right]$ is an intermediate subfield of degree $p$ in the Galois extension $K\left[\sqrt[m_{1}]{N_{1}}, \ldots, \sqrt[m_{i}]{N_{i}}\right] / K$, with Galois group $G=\left\langle\sigma_{1}, \ldots, \sigma_{i}\right\rangle$, where

$$
\sigma_{k}\left(\sqrt[m k]{N_{k}}\right)=\zeta^{m / m_{k}} \sqrt[m k]{N_{k}}, \sigma_{k}\left(\sqrt[m]{N_{j}}\right)=\sqrt[m]{N_{j}}, j \neq k .
$$

Any subgroup of $G$ of index $p$ contains $G^{p}$, so by the Galois correspondence $K\left[\sqrt[p]{N_{i+1}}\right]$ is contained in the fixed field of $G^{p}$, namely $K\left[\sqrt[p]{N_{1}}, \ldots, \sqrt[p]{N_{i}}\right]$. Lemma 1 implies that $N_{1}^{e_{1}} \cdots N_{i}^{e_{i}} N_{i+1} \in K^{p}$ for some $0 \leq e_{i}<p$, against $\mathcal{S}_{p} \cap K^{p}=\emptyset$. Thus $\sqrt[p]{N_{i+1}} \notin K\left[\sqrt[m_{1}]{N_{1}}, \ldots, \sqrt[m_{i}]{N_{i}}\right]$.

Assume if possible, that $4 \mid m_{i+1}$ and $\sqrt{-N_{i+1}} \in K\left[\sqrt[m_{1}]{N_{1}}, \ldots, \sqrt[m_{i}]{N_{i}}\right]$. Then the above argument yields $N_{1}^{e_{1}} \cdots N_{i}^{e_{i+1}} N_{i+1} \in-K^{2}$ for some $0 \leq i<p$. But $-K^{2}=K^{2}$, so $\mathcal{S}_{p} \cap K^{p}=\emptyset$ is violated. This shows $\sqrt{-N_{i+1}} \notin K\left[\sqrt[m_{1}]{N_{1}}, \ldots, \sqrt[m_{i}]{N_{i}}\right]$ when $4 \mid m_{i+1}$.

We deduce from (C) that $\left[K\left[\sqrt[m_{1}]{N_{1}}, \ldots, \sqrt[m_{i+1}]{N_{i+1}}\right]: K\right]=m_{1} \cdots m_{i+1}$.

Lemma 8. Let $n_{1}, \ldots, n_{\ell} \in \mathbb{N}$ and $p$ a prime such that $c(F) \neq p$ and $m_{i}=p^{n_{i}}$ for all $i \in J$. Let $K=F[\zeta]$, where $\zeta$ is a primitive $m$ th root of unity, $m=\operatorname{lcm}\left\{m_{1}, \ldots, m_{\ell}\right\}$. Suppose that $\mathcal{S}_{p} \cap D^{p}=\emptyset$ and $\left|\mathcal{S}_{p} \cap K^{p}\right|=1$, say $M=N_{1}^{f_{1}} \cdots N_{\ell}^{f_{\ell}}$, where $0 \leq f_{i}<p$, and $M^{\sharp}=\left\{i \in J \mid f_{i}=1\right\}$ is nonempty. For $i \in M^{\sharp}$, set $V_{i}=\left\{\sqrt[m_{1}]{N_{1}}, \ldots, \sqrt[m_{\ell}]{N_{\ell}}\right\} \backslash\left\{\sqrt[m_{i}]{N_{i}}\right\}$ and let $m[i]$ be the product of all $m_{j}$ with $j \neq i$. Then

(a) $\left[K\left[V_{i}\right]: K\right]=m[i]$ and $\sqrt[p]{N_{i}} \notin F\left[V_{i}\right]$ for all $i \in M^{\sharp}$.

(b) If $p$ is odd or $m_{i}=2$ for at least one $i \in M^{\sharp}$, then (1) holds.

(c) If $4 \mid m$ and $\mathcal{S}_{2} \cap\left(-D^{2}\right)=\emptyset$, then $\sqrt{-N_{i}} \notin F\left[V_{i}\right]$ for all $i \in M^{\sharp}$, so (1) holds.

(d) If $4 \mid m_{i}$ for all $i \in M^{\sharp}$ and $M \in \mathcal{S}_{2} \cap\left(-D^{2}\right)$, say $M=-d^{2}$ with $d \in D$, then (11) holds if and only if given any $i \in M^{\sharp}$, (4) fails.

Proof. Let $i \in M^{\sharp}$. By Lemma 7, we have $\left[K\left[V_{i}\right]: K\right]=m[i]$ and hence $\left[F\left[V_{i}\right]: F\right]=m[i]$. Suppose, if possible, that $\sqrt[p]{N_{i}} \in F\left[V_{i}\right]$ and set $Y_{i}=\{\zeta\} \cup V_{i}$. Then $F\left[\sqrt[p]{N_{i}}\right]$ is an intermediate subfield of degree $p$ in the Galois extension $F\left[Y_{i}\right] / F$, with Galois group $G=H \rtimes U$, where $H=\left\langle\sigma_{j} \mid j \neq i\right\rangle$ is the Galois group of $F\left[Y_{i}\right] / F[\zeta]$ and each $\sigma_{k}$ is as in the proof of Lemma 7, and $U$ is the Galois group of $F\left[Y_{i}\right] / F\left[V_{i}\right]$. The subgroup $S$ of $G$ corresponding to $F\left[\sqrt[p]{N_{i}}\right] \subseteq F\left[V_{i}\right]$ in the Galois correspondence has index $p$ and contains $U$. Therefore $S \supseteq H^{p} \rtimes U$, so $F\left[\sqrt[p]{N_{i}}\right]$ is contained in the fixed field of $H^{p} \rtimes U$, namely $F\left[W_{i}\right]$, where $W_{i}=\left\{\sqrt[p]{N_{1}}, \ldots, \sqrt[p]{N_{\ell}}\right\} \backslash\left\{\sqrt[p]{N_{i}}\right\}$. It follows from Lemma 1 that $N_{1}^{e_{1}} \cdots N_{\ell}^{e_{\ell}} \in F^{p}$, where all $0 \leq e_{j}<p$ and $e_{i}=1$. By the rational root theorem, $F^{p} \cap D=D^{p}$, so $\mathcal{S}_{p} \cap D^{p}=\emptyset$ is violated.

If $m_{i}=2$ for at least one $i \in M^{\sharp}$, then (1) has been established. Likewise, if $p$ is odd, then (1) follows from (C). Suppose next that $4 \mid m$ and $\mathcal{S}_{2} \cap\left(-D^{2}\right)=\emptyset$. We claim that $\sqrt{-N_{i}} \notin F\left[V_{i}\right]$. If not, arguing as above, we see that $-N_{1}^{e_{1}} \cdots N_{\ell}^{e_{\ell}} \in F^{2} \subseteq K^{2}$, where all $0 \leq e_{j}<2$ and $e_{i}=1$. On the other hand, $M \in K^{2}$ and $-1 \in K^{2}$, so $-M \in K^{2}$ and therefore the product of all $N_{j}^{e_{j}+f_{j}}$, with $j \neq i$, must be in $K^{2}$. The uniqueness of $M$ in $\mathcal{S}_{2} \cap K^{2}$ forces $e_{j}=f_{j}$ for all $j \neq i$. Thus $-M \in F^{2}$ and hence $M \in \mathcal{S}_{2} \cap\left(-D^{2}\right)$, a contradiction. Thus (11) follows from (C) in this case as well.

Suppose finally that $4 \mid m_{i}$ for all $i \in M^{\sharp}$ and $M \in \mathcal{S}_{2} \cap\left(-D^{2}\right)$, say $M=-d^{2}$ with $d \in D$. Fix any $i \in M^{\sharp}$ and set $L_{i}=F\left[V_{i}\right]$. It remains to decide when $N_{i} \in-4 L_{i}^{4}$. Since $4 \mid m_{j}$ for all $j \in M^{\sharp}$, the product of all $N_{j}^{f_{j}}$ with $j \neq i$ and $j \in M^{\sharp}$, belongs to $L_{i}^{4}$. Thus

$$
N_{i} \in-4 L_{i}^{4} \Leftrightarrow M \in-4 L_{i}^{4} \Leftrightarrow d^{2} \in 4 L_{i}^{4} \Leftrightarrow \pm 2 d \in L_{i}^{2},
$$

and, by Lemma 1, this happens if and only if (4) holds. 


\section{Proofs of Theorems A And B}

Proof of Theorem A. It is clear that (1) implies $\mathcal{S}_{p} \cap D^{p}=\emptyset$. Suppose $\mathcal{S}_{p} \cap D^{p}=\emptyset$ and let $K=F[\zeta]$, where $\zeta$ is a primitive $m$ th root of unity. By Lemmas 7 and 8 , it suffices to show that $\left|\mathcal{S}_{p} \cap K^{p}\right| \leq 1$. Suppose not and let $M \neq N$ be in $\mathcal{S}_{p} \cap K^{p}$. As $M, N$ have degree $p$ over $F$, we see that $p \mid[K: F]$. By Lemma $4, \operatorname{Gal}(K / F)$ has a unique subgroup of index $p$, so by the Galois correspondence, $K / F$ has a unique intermediate field of degree $p$. We deduce $F[\sqrt[p]{M}]=F[\sqrt[p]{N}]$ and Lemma 1 implies $M N^{i} \in F^{p}$ for some $i \in \mathbb{Z}$. Since $M \neq N$, this is disallowed by $\mathcal{S}_{p} \cap D^{p}=\emptyset$.

Proof of Theorem B. It is clear that $\mathcal{S}_{2} \cap D^{2}=\emptyset$ follows from (1). Suppose $\mathcal{S}_{2} \cap D^{2}=\emptyset$. We will show that (1) holds if and only if $\left(N_{1}, \ldots, N_{\ell}\right)$ is not 2-defective.

By Lemmas 6, 7 and 8, we may restrict to the case when $\left|\mathcal{S}_{2} \cap K\right|=3$, in which case by Lemmas 5 and 6 there is a single element $M \in \mathcal{S}_{2} \cap\left(-D^{2}\right)$, and we necessarily have $-1 \notin F^{2}$ and $8 \mid m$.

Now $-d^{2}=M=N_{1}^{f_{1}} \cdots N_{\ell}^{f_{\ell}}$, where $0 \leq f_{i}<2$ and $M^{\sharp}=\left\{i \in J \mid f_{i}=1\right\}$ is nonempty. Fix any $i \in M^{\sharp}$ and let $S_{2}^{i}$ stand for the analogue of $S_{2}$ corresponding to $\left\{N_{1}, \ldots, N_{\ell}\right\} \backslash\left\{N_{i}\right\}$. By the uniqueness of $M$ in $\mathcal{S}_{2} \cap\left(-D^{2}\right)$, we see that $\mathcal{S}_{2}^{i} \cap\left(-D^{2}\right)=\emptyset$. It follows from Lemma 6 that $\left|\mathcal{S}_{2}^{i} \cap K^{2}\right| \leq 1$

Suppose first that $\left|\mathcal{S}_{2}^{i} \cap K^{2}\right|=0$. Set $V_{i}=\left\{\sqrt[m_{1}]{N_{1}}, \ldots, \sqrt[m_{\ell}]{N_{\ell}}\right\} \backslash\left\{\sqrt[m_{i}]{N_{i}}\right\}$, and let $m[i]$ be the product of all $m_{k}$ such that $k \neq i$. Then $\left[K\left[V_{i}\right]: K\right]=m[i]$ by Lemma 7 . Thus $F\left[V_{i}\right]$ is linearly disjoint from $K$ over $F$. It follows that $\sqrt{-1} \notin F\left[V_{i}\right]$. For if $\sqrt{-1} \in F\left[V_{i}\right]$, then from $-1 \notin F^{2}$ we deduce that $1, \sqrt{-1}$ are $F$-linearly independent elements from $F\left[V_{i}\right]$, and hence $K$ linearly independent elements from $K\left[V_{i}\right]$, which cannot be as $4 \mid m$. Since $M \in-D^{2}$, we have $F[\sqrt{-1}]=F[\sqrt{M}]$. Thus $\sqrt{M} \notin F\left[V_{i}\right]$ and therefore $\sqrt{N_{i}} \notin F\left[V_{i}\right]$. If there is some $i \in M^{\sharp}$ such that $m_{i}=2$, this shows that (1) holds. If, on the other hand, $4 \mid m_{i}$ for all $i \in M^{\sharp}$, then (10) holds if and only if (4) fails, as in the proof of Lemma 8.

Suppose next that $\left|\mathcal{S}_{2}^{i} \cap K^{2}\right|=1$ and let $N \in S_{2}^{i} \cap K^{2}$. Note that $N \notin-D^{2}$. We have $N=N_{1}^{g_{1}} \cdots N_{\ell}^{g_{\ell}}$, where $g_{i}=0,0 \leq g_{j}<2$ and $N^{\sharp}=\left\{j \in J \mid g_{j}=1\right\}$ is nonempty. Fix any $j \in N^{\sharp}$ and let $S_{2}^{i, j}$ stand for the analogue of $S_{2}$ corresponding to $\left\{N_{1}, \ldots, N_{\ell}\right\} \backslash\left\{N_{i}, N_{j}\right\}$. It is then clear that $S_{2}^{i, j} \cap K^{2}=\emptyset$. Set $V_{i, j}=\left\{\sqrt[m_{1}]{N_{1}}, \ldots, \sqrt[m_{\ell}]{N_{\ell}}\right\} \backslash\left\{\sqrt[m_{i}]{N_{i}}, \sqrt[m_{j}]{N_{j}}\right\}$, and let $m[i]$ (resp. $\left.m[i, j]\right)$ be the product of all $m_{k}$ such that $k \neq i$ (resp. $\left.k \neq i, j\right)$. Then $\left[K\left[V_{i, j}\right]: K\right]=m[i, j]$ by Lemma 7 . As above, we deduce that $\sqrt{-1} \notin F\left[V_{i, j}\right]$. Since $4 \mid m$ and $S_{2}^{i} \cap\left(-D^{2}\right)=\emptyset$, Lemma 8 ensures that $\left[F\left[V_{i}\right]: F\right]=m[i]$ as well as $\sqrt{ \pm N_{j}} \notin F\left[V_{i, j}\right]$. We deduce from Lemma 2 that $\sqrt{-1} \notin F\left[V_{i}\right]$. The rest of the argument follows as in the above case.

\section{Primitive Elements}

Isaacs [I] considered the problem of when $F[\alpha, \beta]=F[\alpha+\beta]$ for algebraic separable elements $\alpha, \beta$ of degrees $m, n$ over $F$. He proved that if $[F[\alpha, \beta]: F]=m n$ (he actually assumed $\operatorname{gcd}(m, n)=1$ but used only the stated condition) but $F[\alpha, \beta] \neq F[\alpha+\beta]$ then $F$ has prime characteristic $p$ and the following conditions hold: $p \mid m n$ or $p<\min \{m, n\}$; if $m, n$ are prime powers, then $p \mid m n ; p$ divides the order of the Galois group of a normal closure of $F[\alpha, \beta]$.

The condition $p<\min \{m, n\}$ was later improved to $p<\min \{m, n\} / 2$ by Diviš [D].

Using Isaacs' result we readily see that $F\left[\sqrt[m_{1}]{N_{1}}, \ldots, \sqrt[m_{\ell}]{N_{\ell}}\right]=F\left[b_{1} \sqrt[m_{1}]{N_{1}}+\cdots+b_{\ell} \sqrt[m_{\ell}]{N_{\ell}}\right]$ for any nonzero $b_{1}, \ldots, b_{\ell} \in F$ in Theorems $\mathrm{A}, \mathrm{B}$ and $\mathrm{C}$, provided the following conditions hold: $c(F) \neq p$ and $\mathcal{S}_{p} \cap D^{p}=\emptyset$ in Theorem A; $c(F) \neq 2, \mathcal{S}_{2} \cap D^{2}=\emptyset$, and $\left(N_{1}, \ldots, N_{\ell}\right)$ is not 2-defective in Theorem B; $c(F) \nmid m \varphi(m)$ (Euler's function), $\mathcal{S}_{2} \cap D^{p}=\emptyset$ for all $p \in \mathcal{P}$, and $\left(N_{i(2,1)}, \ldots, N_{i(2, \ell(2))}\right)$ is not 2-defective in Theorem C.

It is possible that $F[\alpha, \beta] / F$ be a finite Galois extension, $[F[\alpha, \beta]: F]=[F[\alpha]: F][F[\beta]: F]$ and still $F[\alpha, \beta] \neq F[\alpha+\beta]$. A family of examples can be found in CS, Example 2.3]. 


\section{REFERENCES}

[A] T. Albu, Kummer extensions with few roots of unity, J. Number Theory 41 (1992) 322-358.

[B] A.S. Besicovitch, On the linear independence of fractional powers of integers, J. London Math. Soc 15 (1940) 3-6.

[CS] L. Cagliero and F. Szechtman, On the theorem of the primitive element with applications to the representation theory of associative and Lie algebras, Canad. Math. Bull. 57 (2014) 735-748.

[C] A. Capelli, Sulla riduttibilitá delle equazioni algebriche, Nota prima, Rend. Accad. Sci. Fis. Mat. Soc. Napoli 3 (1897) 243-252.

[CO] R. Carr and C. O'Sullivan, On the linear independence of roots, Int. J. Number Theory (2009) 161-171.

[D] B. Diviš, On the degrees of the sum and product of two algebraic elements, Number Theory and Algebra, 19-27, Academic Press, New York, 1977.

[F] H. Flanders, [Solution to a problem proposed by D.J. Newman], Amer. Math Monthly 67 (1960) 188-189.

[H] H. Hasse, Klasssenkörpertheorie, Mimeographed lectures, Marburg 1932-33, 187-195.

[I] I.M. Isaacs, Degrees of Sums in a Separable Field Extension, Proceedings of the American Mathematical Society 25 (1970) 638-641

[J] Z. Jian-Ping, On the degree of extensions generated by finitely many algebraic numbers, J. Number Theory 34 (1990) 133-141.

[M] L.J. Mordell, On the linear independence of algebraic numbers, Pacific J. 3 (1953) 625-630.

[R] L. Rédei, Algebra, Volume 1, Pergamon Press, Oxford, 1967.

[Ri] I. Richards, An application of Galois theory to elementary arithmetic, Adv. in Math. 3 (1974) 268-273.

[Ro] R.L. Roth, On extension of $\mathbb{Q}$ by square roots, Amer. Math Monthly 78 (1971) 382-393.

[S] C.L. Siegel Algebraische Abhängigkeit von Wurzein, Acta Arith. 21 (1972) 59-64.

[U] H.D. Ursell, The degree of radical extensions, Canad. Math. Bull. 17 (1974) 615-617.

[V] K. T. Vahlen, Über reductible Binome, Acta Math. 19 (1895) 195-198.

[Vi] I.M. Vinogradov, Elements of number theory, Dover, New York, 2016.

Department of Mathematics and Statistics, University of Regina, Canada

Email address: fernando.szechtman@gmail.com 